\title{
Predictive Factors in Locally Advanced Rectal Cancer Treated With Preoperative Hyperfractionated and Accelerated Radiotherapy
}

\author{
HANIFA BOUZOURENE, MD, FRED T. BOSMAN, MD, PHD, \\ MAURICE MATTER, MD, AND PHILIPPE COUCKE, MD
}

\begin{abstract}
This study examines the prognostic significance of pathologic factors in patients with primary locally advanced rectal cancer treated prospectively with preoperative radiotherapy. From 1992 to 1998,104 patients with rectal cancer of grades $\mathrm{T} 3$ or $\mathrm{T} 4$ and any $\mathrm{N}$ underwent preoperative radiotherapy followed by surgical resection. Survival curves were estimated according to the Kaplan-Meier method. Correlation of outcome with clinicopathologic variables (pathologic tumor and lymph node staging, histology, radial resection margin [RRM], clearance, vessel involvement, and tumor regression grade [TRG], quantitated in 5 grades) was evaluated using the Cox proportional hazards model. None of the patients achieved a histologically confirmed complete pathologic response, but $79 \%$ of the patients showed partial tumor regression (TRG2-4) and $21 \%$ did not show any tumor regression (TRG5). Among the tumors, $22 \%$ were of a mucinous type. The RRM was free of tumor in $76 \%$ of the surgical specimens. The median clearance was $2 \mathrm{~mm}$. Vascular invasion was present in 37 cases $(36 \%)$. In the univariate analysis, lymph node metastases, absence of tumor regression, positive RRM, and vascular invasion were correlated with adverse overall survival and disease-
\end{abstract}

Colorectal cancer is a significant cause of morbidity and mortality in Western populations. More than $1 / 3$ of cases occur in the rectum, with an outcome after surgery less favorable than for colon cancer, as reflected in local recurrence rates of $20 \%$ to $50 \%$ after traditional surgical treatment. ${ }^{1}$ Moreover, rectal cancer is characterized by a higher incidence of regional lymph nodes and distant metastases. Preoperative radiotherapy has been shown to reduce local failure rates and to improve overall survival. ${ }^{1-4}$ However, results of different clinicopathologic studies are sometimes contradictory, which might be related to the heterogeneity in radiation treatment-related factors and clinical stages. Absence of a standardized method for pathologic analysis might be another source of discrepancies.

We have adopted a treatment schedule that aims to accelerate the treatment to counter tumor proliferation during treatment and to reduce the risk of late complications by hyperfractionation (ie, reducing the dose

From the Institute of Pathology, Lausanne, Switzerland and the Departments of Surgery and Radiation-Oncology, Centre Hospitalier Universitaire Vaudois, Lausanne, Switzerland. Accepted for publication February 11, 2003.

Address correspondence and reprint requests to Dr. Hanifa Bouzourene, Institute of Pathology, Bugnon 25, CH 1011, Lausanne, Switzerland.

(c) 2003 Elsevier Inc. All rights reserved.

0046-8177/03/3406-0010\$30.00/0

doi:10.1016-S0046-8177(03)00176-X free survival; absence of tumor regression, positive RRM, and clearance $<2 \mathrm{~mm}$ were correlated with local recurrences; and advanced pT stage was correlated only with disease-free survival. However, in the multivariate analysis, only lymph node metastases and RRM were independent prognostic factors for overall survival and disease-free survival, and clearance $<2 \mathrm{~mm}$ was an independent prognostic factor for local control. Pathologic parameters remain strong determinants of local recurrence and survival in locally advanced rectal cancer, treated preoperatively with hyperfractionated and accelerated radiotherapy. We show that patients with advanced pT, positive lymph nodes, vascular invasion, positive RRM, clearance $<2 \mathrm{~mm}$, or absence of tumor regression are known to have poor clinical outcome. Hum Pathol 34:541-548. @ 2003 Elsevier Inc. All rights reserved.

Key words: preoperative radiotherapy; cancer; rectum.

Abbreviations: CT, computed tomography; DFS, disease-free survival; HART, hyperfractionated accelerated radiotherapy; OS, overall survival; RRM, radial resection margin; TRG, tumor regression grade.

per fraction). This hyperfractionated accelerated radiotherapy (HART) schedule was tested in locally advanced rectal cancer, that is, T3 and T4 and any $\mathrm{N}$, or any $\mathrm{T}$ but $\mathrm{N}+$, rectal cancers. ${ }^{5,6}$ We demonstrated that preoperative HART is feasible and is associated with a lower toxicity than postoperative radiotherapy. ${ }^{5}$ Based on this initial experience, a phase II trial was initiated.

Whether or not classical predictive factors of survival for colorectal cancer are still important after neoadjuvant therapy is a question frequently asked by pathologists, and only sporadic studies with a systematic pathologic approach try to answer it. In this study we attempted to correlate response to radiotherapy with pathologic variables and to determine which pathologic variables, including histological assessment of response to radiotherapy, correlated with disease outcome.

\section{PATIENTS AND METHODS}

The study group comprised 104 patients with locally advanced rectal cancer recruited in a phase II trial (93-01) on preoperative HART between 1992 and 1998. Assessment of the clinical stage was based on digital rectal examination, completed by both rectal ultrasonography and computed tomography (CT) scan. During this time period, all patients were eligible if they presented at preoperative work-up with cT3 or cT4, regardless of the N stage. The HART protocol was reviewed by the local ethics committee. All patients gave informed consent. Patients with coexisting cancer or chronic 
inflammatory bowel disease were excluded from this study. Most of the patients (90) underwent surgery in the Department of Surgery, Hospital Center of the University of Vaudois at Lausanne. Abdominoperineal resection was performed in 52 patients; low anterior resection, in 50 patients; and abdominal transanal resection, in 2 patients. Although surgeons claim that total mesorectal excision is a standard surgical approach, we do not have the ability to assess whether this surgical approach has been applied systematically. Patient age ranged from 28 to 85 years (median, 63 years), and the female/male ratio was 1.2. Before initiation of treatment, all patients underwent complete clinical examination, endoscopic biopsy, blood count, renal and hepatic function assessment, and carcinoembryonic antigen determination. Distant metastatic disease was excluded by chest x-ray and abdominal ultrasound or CT scan. Assessment of the local tumor extension was done by digital rectal examination, rectal ultrasound, or CT scan.

According to cTNM staging based on digital rectal examination, rectal ultrasound, or CT scan, ${ }^{7} 104$ patients in this cohort were clinically classified as stage T3-T4, and 27 were $\mathrm{N}+$. Only 10 of the tumors were mobile, and 74 were fixed or tethered. The status of the remaining tumors in terms of fixity versus mobility was unknown. All patients were irradiated with a linear accelerator with a minimal accelerating potential of 6 $\mathrm{mV}$. The dose per fraction was $1.6 \mathrm{~Gy}$ (26 fractions), the interfraction interval was at least 6 hours, and the total dose was $41.6 \mathrm{~Gy}$, given over 2.5 weeks. The time lapse between the end of radiotherapy and surgical resection in most cases was within 6 days (median, 5 days). Four patients who had a positive resection margin and thus had no curative resection (R1) received additional adjuvant chemotherapy after surgery.

\section{Macroscopic Assessment}

The surgical specimens were opened through the anterior wall and fixed in $10 \%$ buffered neutral formalin for 24 hours. The external surface of specimen was painted with India ink to facilitate recognition of the circumferal margin, also known as the radial resection margin (RRM). The entire tumor and attached mesorectum were serially sliced at 3- to 4-mm intervals perpendicular to the longitudinal axis of the rectum, which allowed macroscopic identification of the areas of deepest invasion. These were sampled for histological confirmation. Tumors $<5 \mathrm{~cm}$ in maximal dimension were included in toto. For assessment of perirectal lymph nodes, the mesorectal fat was removed after tumor sampling and cleared in a carnoy solution for 24 hours, and all macroscopically identifiable lymph nodes were submitted for histological examination.

\section{Histological Assessment}

Tissue samples were embedded in paraffin, cut, and stained with hematoxylin and eosin. All rectal tumors were retrospectively reanalyzed by 1 of the authors (B.H.). The tumors were classified according to the World Health Organization classification system for tumors of the digestive system and staged according to the TNM classification system. ${ }^{7,8}$ Tumor staging was based on the deepest infiltration of the epithelial component of the cancer. An adenocarcinoma was considered to be of the mucinous type if $>50 \%$ of the tumor was composed of mucin. The presence of tumor cells in lakes of mucin was a prerequisite for a diagnosis of lymph node metastatic mucinous carcinoma and for tumor staging. When lakes of mucin found in the deepest part of a tumor were devoid of tumor cells, they were not considered in the tumor staging. When needed, staining with cytokeratins was performed to detect isolated tumor cells into lakes of mucus. Tumor downstaging was obtained by comparison of the cT and $\mathrm{pT}$ stages.

Tumor regression was graded according to a method described by Mandard et $\mathrm{al}^{9}$ for the assessment of pathologic response after neoadjuvant chemoradiotherapy in esophageal carcinomas and already used by our group in a previous study on a series of rectal cancer treated with preoperative radiotherapy. ${ }^{10}$ This method distinguishes 5 tumor regression grades (TRGs), based on the presence of residual tumor cells and the extent of fibrosis. TRG1 is defined as absence of residual cancer and fibrosis extending through the different layers of the rectal wall. TRG2 is characterized by rare residual cancer cells scattered throughout the fibrosis (Fig 1A). TRG3 has more residual tumor cells, but fibrosis still predominates (Fig 1B). In TRG4, residual cancer cells predominate in the fibrosis (Fig 1C), and in TRG5, the tumor shows no signs of regression (Fig 1D). Thus fibrosis induced by radiotherapy was not included in the assessment of the tumor infiltration, but it was used as a parameter of downstaging.

The clearance (ie, free distance between the tumor and RRM) was measured on the sections in millimeters. Distal and proximal resection margins, RRM, and the presence or absence of lymphatic or venous invasion were also recorded. No distinction was made between venous or lymphatic involvement by tumor and between extramural or intramural venous invasion

\section{Statistical Analysis and Follow-Up}

All patients were followed for local recurrence and distant metastasis every 6 months for the first 2 years and every year thereafter. A physical examination, serum carcinoembryonic antigen assay, chest x-ray, abdominal ultrasound or CT scan, and pelvic CT scan were included in the follow-up. The events were death (all causes of death included) for overall survival, distant metastases or locoregional relapse or death for disease-free survival, and local or locoregional relapse for local control. Patients who died without local or locoregional relapse were censored at time of death.

All statistical analyses were conducted using the JMP 3.2.2 JUMP software (SAS Institute, Cary, NC). The $\chi^{2}$ test was used for the tests of correlation, and a $P$ value $<0.05$ was considered statistically significant. In univariate and multivariate analysis, overall survival, disease-free survival, and local control were used as the endpoints. In univariate analysis, survival curves were estimated according to the Kaplan-Meier method for the following pathologic variables: $\mathrm{pT}, \mathrm{pN}$, histology, TRG, clearance, proximal and distal margins, RRM, and vascular invasion. The significance of the differences was estimated by the log-rank test. To increase the number of patients per group, the categories of the different pathologic variables were also combined for these analyses, that is, pT1-2 versus pT3-4, well and moderately differentiated adenocarcinomas versus poorly differentiated and mucinous carcinomas, and the responder group (TRG2-4) versus the nonresponder group (TRG5).

Multivariate survival analysis according to the Cox proportional hazards model was constructed by backward elimination of variables reaching a $P$ value of 0.1 in the univariate analysis. Thus the following parameters were selected for OS and DFS: $\mathrm{pT} 1-2$ versus $\mathrm{pT} 3-4, \mathrm{pN} 0$ versus $\mathrm{pN}$ positive, responder group (TRG2-4) versus nonresponder group (TRG5), RRM free, and vascular invasion. In comparison, the following parameters were used for local control: responder 

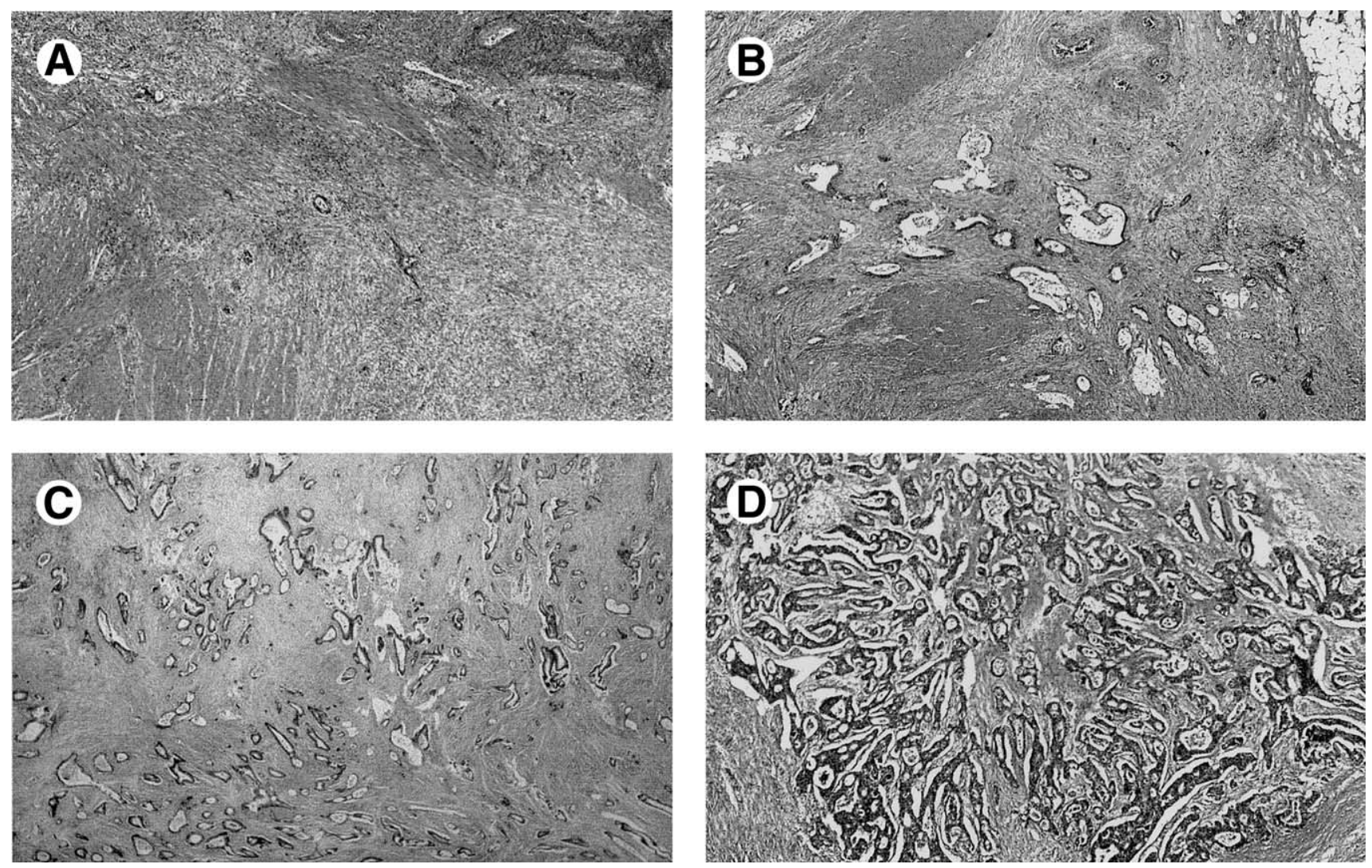

FIGURE 1. Tumor regression assessment of rectal carcinoma after preoperative radiotherapy. (A) Tumor showing rare residual cancer cells scattered through the fibrosis (TRG2). (B) Tumor showing more residual tumor cells, but with fibrosis still predominating (TRG3). (C) In TRG4, there are more residual cancer cells than fibrosis. (D) In TRG5, the tumor shows no signs of regression.

group versus nonresponder group, RRM free, and clearance $<2 \mathrm{~mm}$. Patient age and sex were not included in the multivariate analysis, because these factors were not found to be significantly associated with the aforementioned endpoints.

\section{RESULTS}

\section{Pathologic Findings}

All of the pathologic parameters analyzed are summarized in Table 1 . Of the 104 rectal cancer cases, 2 were pT1 $(2 \%), 21$ were pT2 $(20 \%), 67$ were pT3 $(64 \%)$, and 14 were pT4 (14\%). Of all patients, 44 had a pT stage lower than the preoperatively assessed $\mathrm{T}$ stage ( $43 \%$ of downstaging). Regional lymph node metastases were found in 55 patients $(53 \%)$, but no nodal downstaging was seen. Of the tumors, $25 \%$ were welldifferentiated, $40 \%$ were moderately differentiated, and $13 \%$ were poorly differentiated adenocarcinomas; the remaining 22\% were mucinous carcinomas. Mucinous carcinomas were most often found in patients with pT3-4 $(P=0.04)$.

None of the 104 tumors showed complete tumor regression (TRG1). Partial tumor regression (TRG2-4) was noted in $79 \%$ of the tumors, and no regression (TRG5) was seen in $21 \%$ of the tumors. Of the regressing tumors, $20 \%$ were TRG2, 39\% were TRG3, and 20\% were TRG4. Advanced pathologic stages (pT3-4) fre- quently showed no tumor regression (TRG5) $(P=$ $0.01)$.

Median clearance was $2 \mathrm{~mm}$. The total number of patients with a clearance of $<2 \mathrm{~mm}$ was $54(52 \%)$. Proximal and distal margins were free of tumor in all patients but 1 , in whom both the distal margin and the RRM were positive. A positive RRM was found in 25 (24\%) of all specimens. The other 79 patients $(76 \%)$ were considered to have had a curative resection (R0). In 37 cases $(36 \%)$, lymphatic invasion (23 cases) or venous invasion (14 cases) was found.

\section{Survival and Local Control}

During follow-up (median, 40 months), 29 patients developed distant metastasis $(28 \%)$ and 8 developed local recurrence $(7.7 \%)$. Forty-five patients died, 34 from cancer. The median overall survival (OS) was 54 months, and the median disease-free survival (DFS) was 50 months. The actuarial locoregional recurrence rates were $6.4 \%$ at 2 years and $7.6 \%$ at 5 years. Tumor downstaging did not correlate with survival. The type of surgical resection did not have any influence on survival or local control. Patient age and sex and tumor differentiation did not correlated with outcome. The correlation between the various pathologic variables and outcome parameters are summarized in Table 2. 
TABLE 1. Distribution of Histological Parameters in 104 Postirradiated Rectal Cancers

\begin{tabular}{lrr}
\hline Histological parameters & $\mathrm{n}$ & $\%$ \\
\hline Pathologic staging & & \\
pT1 & 2 & $2 \%$ \\
PT2 & 21 & $20 \%$ \\
pT3 & 67 & $64 \%$ \\
pT4 & 14 & $14 \%$ \\
PN 0 & 49 & $47 \%$ \\
PN + & 55 & $53 \%$ \\
Tumor differentiation & & \\
Well differentiated & 26 & $25 \%$ \\
Moderately differentiated & 42 & $40 \%$ \\
Poorly differentiated & 13 & $13 \%$ \\
Mucinous carcinoma & 23 & $22 \%$ \\
TRG & & \\
1 & 0 & $0 \%$ \\
2 & 20 & $39 \%$ \\
3 & 41 & $20 \%$ \\
4 & 21 & $21 \%$ \\
5 & 22 & $24 \%$ \\
RRM & & $76 \%$ \\
Positive & 25 & $64 \%$ \\
Negative & 79 & $36 \%$ \\
Vascular invasion & & \\
Absent & 67 & \\
Present & 37 &
\end{tabular}

\section{Overall Survival}

Median actuarial OS was significantly lower in patients with lymph node metastases $(P=0.001)$. Tumor differentiation was not correlated with survival even if tumors were grouped into well-differentiated and moderately differentiated adenocarcinomas versus poorly differentiated and mucinous carcinomas. There was a trend for histological response (TRG) to correlate with OS $(P=0.06)$. When grouped together, responders (TRG2-4) showed significantly better overall survival than nonresponders (TRG5) $(P=0.02)$ (Fig 2A). OS was worse in patients with a positive RRM $(P=0.001)$ (Fig 2B) and with vascular invasion $(P=0.01)$ (Fig 2C).

\section{Disease-Free Survival}

Median actuarial DFS was significantly lower in patients with advanced pT stages (pT3-4) $(P=0.03)$ and with lymph node metastases $(P=0.0004)$. The DFS was longer in responders (TRG2-4) than in nonresponders (TRG5) $(P=0.03)$. Moreover, a significant correlation was found between the different grades of tumor response and DFS $(P=0.04)($ Fig $3 \mathrm{~A})$. DFS was also highly correlated with a positive RRM $(P=0.0007)$ (Fig 3B) and vascular invasion $(P=0.005)$ (Fig 3C).

\section{Local Control}

The local control was better in responders (TRG2TRG4) than in nonresponders (TRG5) $(P=0.02)$ (Fig $4 \mathrm{~A})$. Five of the patients with local recurrence had a positive RRM, and all of them had a clearance less than $2 \mathrm{~mm}$. The risk of local recurrence strongly correlated with a positive RRM $(P=0.001)$ (Fig $4 \mathrm{~B})$ and a clearance $<2 \mathrm{~mm}(P=0.005)$ (Fig $4 \mathrm{C})$.

\section{Multivariate Analysis}

The results of multivariate analysis are summarized in Table 3. Lymph node metastases and free RRM were independent prognostic factors for OS and DFS, whereas only clearance $<2 \mathrm{~mm}$ remained an independent prognostic factor for local control.

\section{DISCUSSION}

Both local recurrence and distant metastasis after surgery remain major problems in the curative approach of rectal cancer. Local recurrence is often associated with inadequate tumor resection. ${ }^{11}$ Major im-
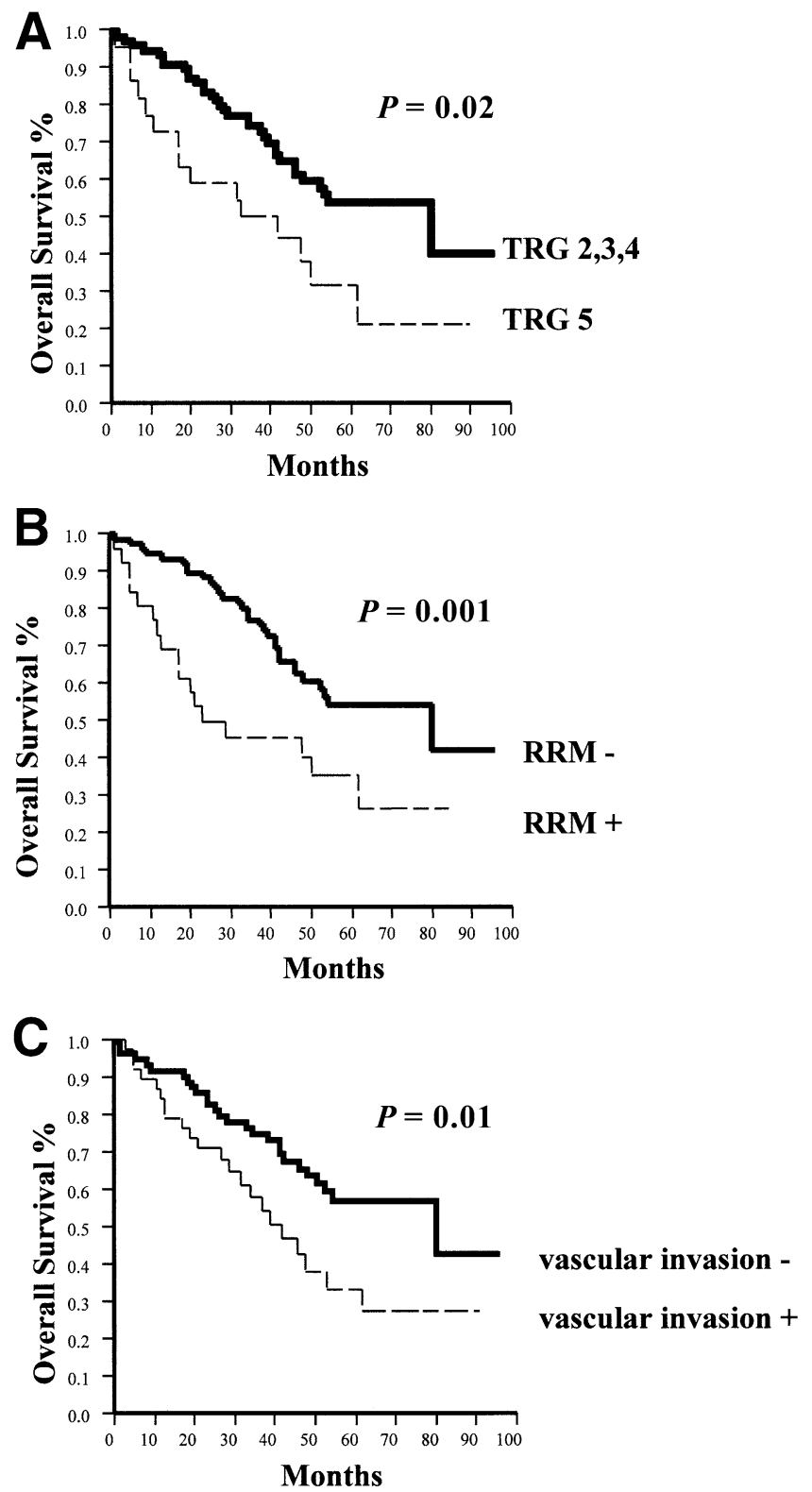

FIGURE 2. OS of TRG, RRM, and vascular invasion studied by univariate analysis in a series of 104 patients with primary locally advanced rectal cancer treated with preoperative radiotherapy. 


\section{A}

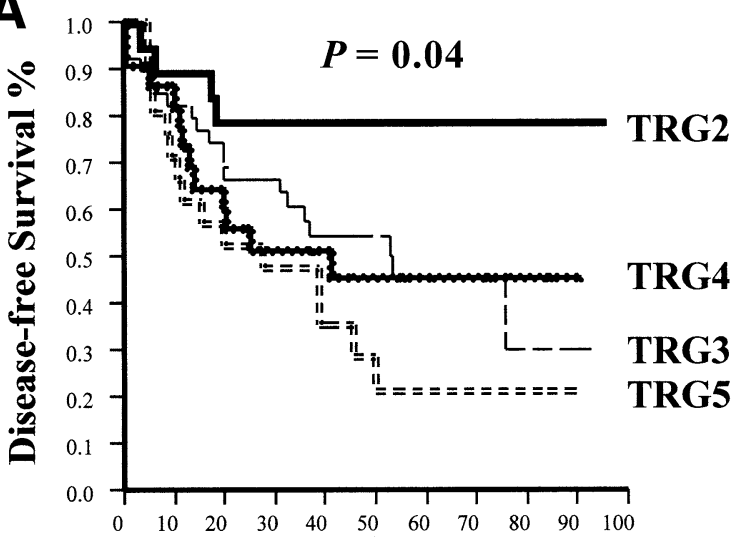

Months

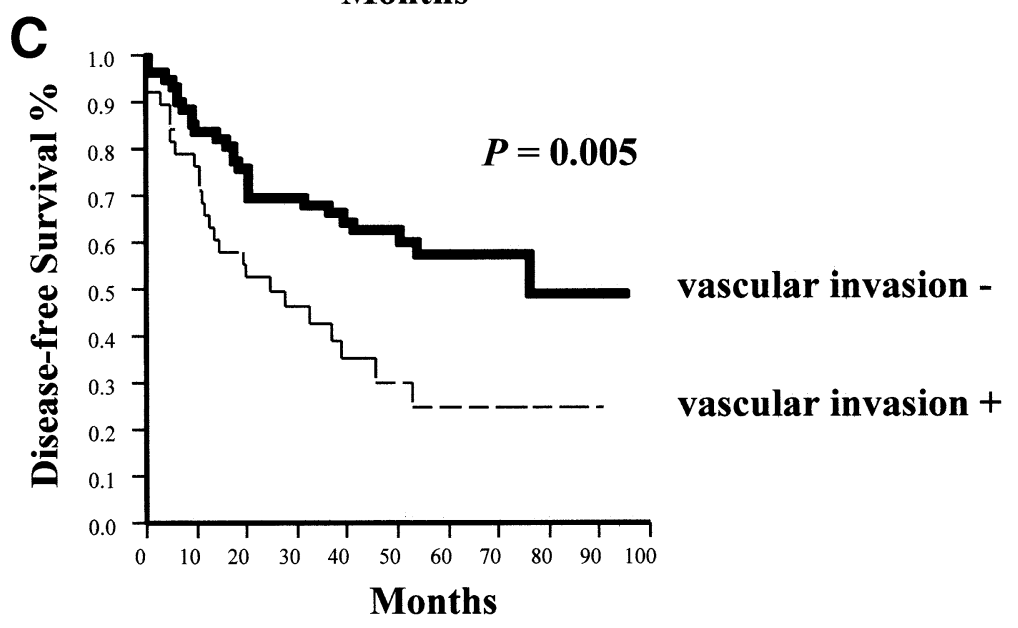

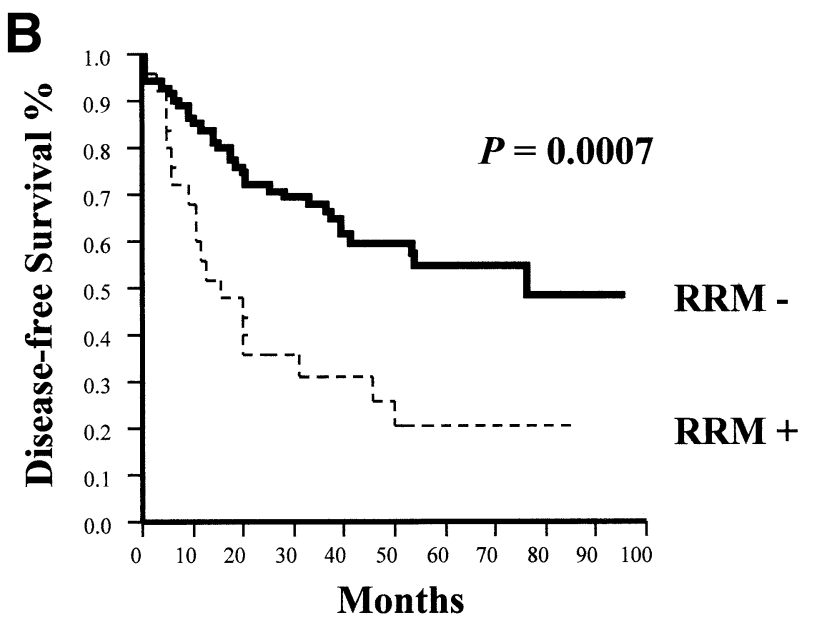

FIGURE 3. DFS of TRG, RRM, and vascular invasion studied by univariate analysis in a series of 104 patients with primary locally advanced rectal cancer treated with preoperative radiotherapy.

provements in the surgical control of local disease have been made since the introduction of total mesorectal excision. ${ }^{12-14}$ Nevertheless, the incidence of local recurrence remains high $(20 \%$ to $40 \%) .2,15$ Randomized studies have shown that preoperative radiotherapy decreases the risk of recurrences. ${ }^{13,15-22}$ Nonetheless, it must be mentioned that most other series included different Dukes' stages, hindering comparison with our

TABLE 2. Correlation Between Histological Parameters and Survival and Local Control in a Series of 104 Patients With Rectal Cancer Treated With Preoperative Radiotherapy

\begin{tabular}{|c|c|c|c|}
\hline Histological parameter & OS & DFS & Local control \\
\hline pT1-2 versus pT3-4 & p 0.1 & p 0.03 & $\mathrm{p} 0.7$ \\
\hline pN stage & p 0.001 & p 0.0004 & $\mathrm{p} 0.4$ \\
\hline Tumor differentiation & $\mathrm{p} 0.2$ & $\mathrm{p} 0.4$ & $\mathrm{p} 0.6$ \\
\hline TRG 2-4 versus TRG5 & p 0.02 & p 0.03 & p 0.02 \\
\hline Positive RRM & p 0.001 & $\mathrm{p} 0.0007$ & p 0.001 \\
\hline clearance $<2 \mathrm{~mm}$ & p 0.9 & $\mathrm{p} 0.7$ & p 0.005 \\
\hline Vascular invasion & p 0.01 & $\mathrm{p} 0.001$ & $\mathrm{p} 0.2$ \\
\hline
\end{tabular}

NOTE: $P$ value $<0.05$. series, which included only locally advanced rectal cancers.

The effect of preoperative radiotherapy on survival is still controversial, with some studies showing a minimal impact $^{17,23}$ and others a significant impact. ${ }^{13,15,18,19,21}$ The discrepancies among trials may be due to heterogeneity in radiation treatment-related factors and differences in clinical stages, as well as to absence of a standardized method for pathologic analysis. Whether or not classical predictive factors of survival for colorectal cancer are still important after neoadjuvant therapy is a question frequently asked by pathologists, and only sporadic studies with a systematic pathologic approach have attempted to answer it. A systematic pathologic workup is essential to compare the results of preoperative radiotherapy among different groups. We have reported previously that preoperative HART, an innovative schedule of radiotherapy in rectal cancer, is feasible, improves the radical resection rate, and induces a significantly lower acute toxicity compared with postoperative radiotherapy. 5,6

The aim of the present prospective study was to evaluate the histological factors of prognostic value in a series of patients with locally advanced rectal cancer 
A

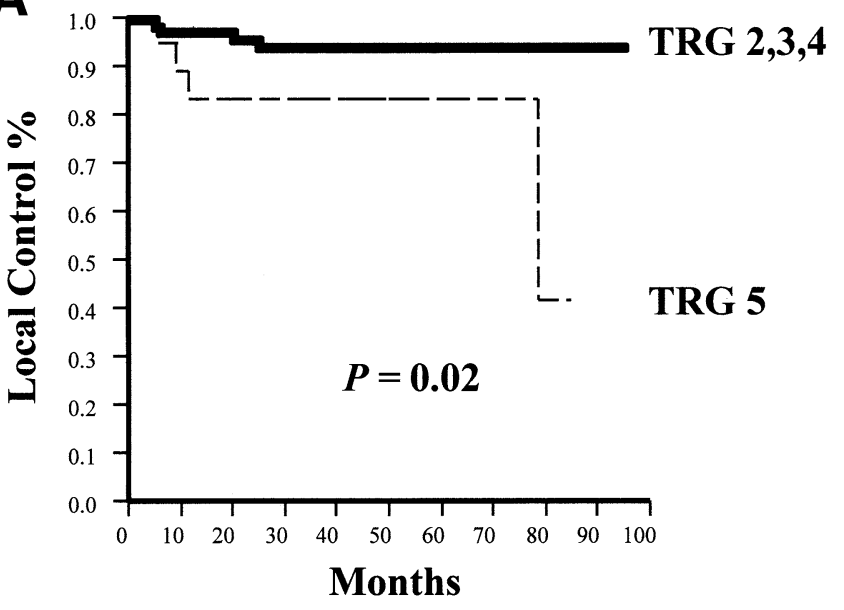

B

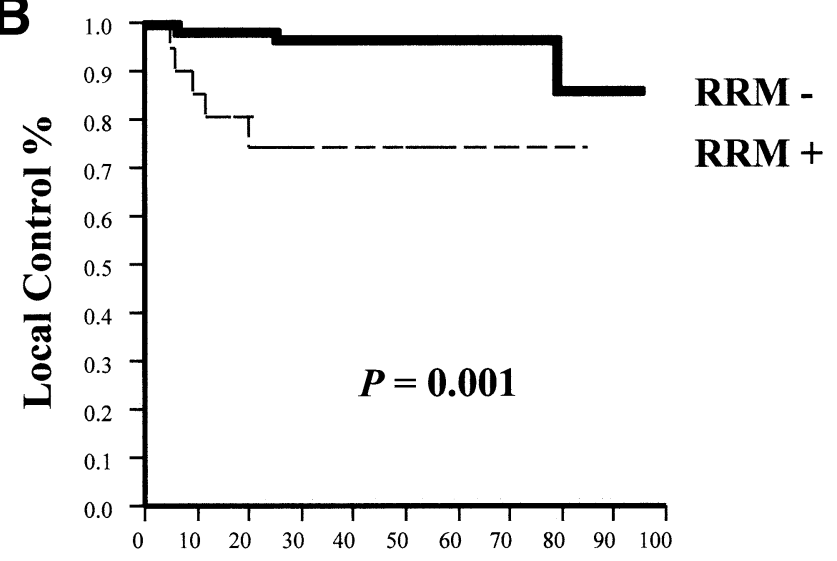

Months

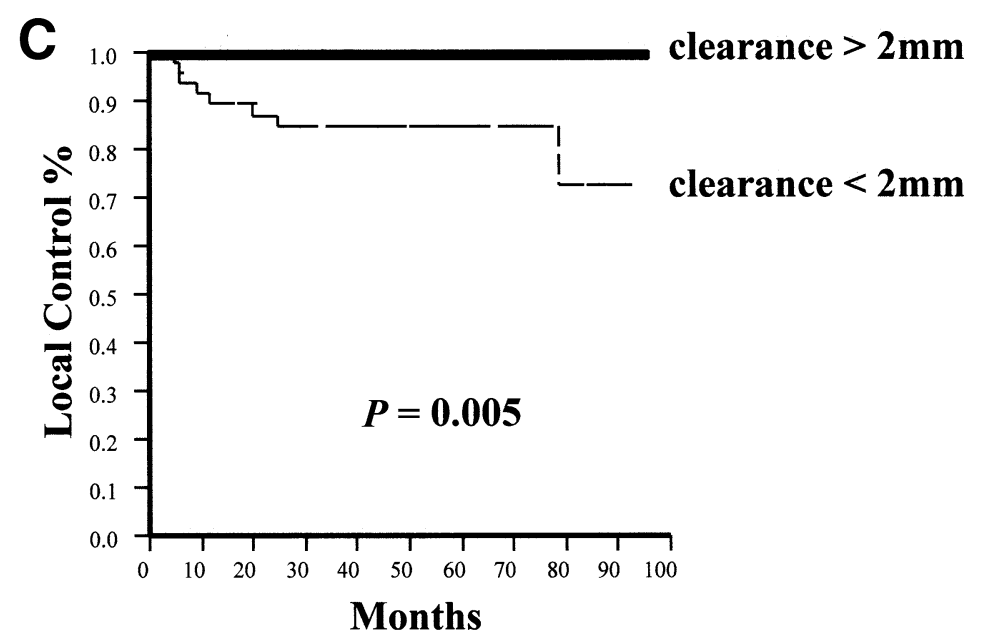

FIGURE 4. Local control of TRG, RRM, and clearance studied by univariate analysis in a series of 104 patients with primary locally advanced rectal cancer treated with preoperative radiotherapy.

treated with a preoperative HART schedule. To this end, we ensured meticulous standardization of specimen processing. We first paid attention to factors known to adversely influence the risk of local recurrence or survival in rectal cancer treated with surgery alone, including such tumor characteristics as tumor stage, differentiation grade, tumor type, lymphatic or venous invasion, RRM, and clearance. ${ }^{11,24,25}$ We also looked at the prognostic role of tumor regression in rectal cancers preoperatively treated by HART. We found in this study, as in a previously reported study, ${ }^{10}$ that advanced $\mathrm{pT}$ was associated with a decreased DFS. As expected, lymph node metastases correlated with adverse outcome. ${ }^{26-28}$ We observed a higher incidence of positive lymph nodes on pathologic examination than by clinical staging. This finding is in disagreement with some reports ${ }^{1,4,29,30}$ but in agreement with others indicating a higher or unchanged number of lymph node metastases. ${ }^{17,27,31,32}$ Our results underline the lack of reliability of preoperative staging by radiologic techniques and the need for meticulous analysis of lymph nodes, which we attained by clearing the mesorectum.
In our series we found a mucinous carcinoma rate of $22 \%$, which is less than the rate of $33 \%$ reported in 1 series of nonirradiated rectal cancer. ${ }^{33}$ However, a study by Nagtegaal et $\mathrm{al}^{34}$ comparing irradiated and nonirradiated rectal carcinomas found that the irradiated group had significantly more mucinous carcinomas. The authors hypothesized that irradiation might induce changes in the consistency of mucin, causing tumor expansion. However, in this case, one would expect a shift to increased mucin in all tumors, which

TABLE 3. Multivariate Analysis in a Series of 104 Patients With Rectal Cancer Treated With Preoperative Radiotherapy

\begin{tabular}{lccc}
\hline \multicolumn{1}{c}{ Parameter } & Relative risk & Confidence interval & $P$ value \\
\hline OS & & & \\
$\quad$ Lymph nodes positive & 2.60 & $1.35-5.38$ & 0.004 \\
$\quad$ RRM free & 0.68 & $0.50-0.93$ & 0.02 \\
DFS & & & \\
$\quad$ Lymph nodes positive & 3.39 & $1.69-7.53$ & 0.0004 \\
RRM free & 0.66 & $0.48-0.90$ & 0.01 \\
\hline
\end{tabular}


was not observed. Further research on the relationship between radiation and the induction of mucinous carcinoma is merited.

In our series, the rate of poorly differentiated carcinomas was higher than the rate reported in a series of nonirradiated rectal carcinomas (13\% versus 5\%). ${ }^{35}$ However, our result is in accordance with that of previously reported study of rectal cancer treated with preoperative short-term radiation regimes, ${ }^{2}$ but not with a trial using conventional long-term preoperative radiotherapy schedules. ${ }^{4}$ This finding is difficult to explain, but it might suggest that the differentiated component of a carcinoma is more radiosensitive and that the poorly differentiated component persists after irradiation. However, even if we had observed a shift toward a more important proportion of poorly differentiated and mucinous carcinomas, these changes would have no impact on survival, because we did not find any correlation between tumor type and survival.

The significance of the presence of blood and lymphatic vessel invasion after radiotherapy remains unknown. We found in our study that vascular invasion significantly decreased OS and DFS. The extent of blood vessel invasion could be a criterion for selecting patients with rectal cancer who may benefit from adjuvant therapy. However, this finding needs to be confirmed by other studies.

The present study demonstrates the importance of the RRM in the survival of patients with locally advanced rectal cancer treated preoperatively with radiotherapy. The RRM was free of tumor in $76 \%$ of the surgical specimens but was involved in $24 \%$ of the patients, who thus had no curative resection. Although we cannot exclude the possibility that nonstandard surgery with a total mesorectal procedure is at the origin of the high positive RRM rate, we can tentatively explain this observation by the fact that only locally advanced rectal cancer with a $72 \%$ of initially tethered or fixed tumors were selected in this series, and the entire tumors with their attached mesorectum were systematically sampled with special emphasis to detect positive resection margins. Nevertheless, the actuarial locoregional recurrence rate at 5 years is only $7.6 \%$. The presence of tumor in the radial margin was strongly associated not only with local recurrence, but also with poor survival. Our results compare favorably with the results of the preoperative radiotherapy Swedish Rectal Cancer trial, in which the rate of local recurrence was $11 \% .{ }^{1}$ However, our data cannot be compared directly with data from the Dutch total mesorectal study, in which the local recurrence rate was $2.4 \% .^{14,36}$ There are several reasons for this. First, in the Dutch study there were $30 \%$ of patients with stage I, whereas in our study stage I was an exclusion criteria. Second, the results of the Dutch study are given at 2 years, whereas we are providing actuarial results at 5 years (median follow-up, 40 months). Finally, in the Dutch study there was extensive surgical training, which was not the case in our study, because we were treating patients addressed by a variety of surgeons.

In addition, we found that in patients without RRM involvement but with clearance $<2 \mathrm{~mm}$, the local recurrence rate was higher. In the multivariate analysis, clearance remained an independent parameter for the prediction of local recurrences. Although the determining factor for local control is a free RRM, quality control of the surgical technique by standardized pathologic examination of the specimen is by no means the rule. ${ }^{14}$ Our results indicate that adequate histopathologic assessment not only of RRM, but also of clearance provides clinically relevant parameters. Even in the case of a negative RRM, the risk of local failure remains high if clearance is $<2 \mathrm{~mm}$. Thus precise assessment of RRM as well as clearance can justify further treatment to prevent local recurrence. RRM involvement is also important to the prediction of distant metastases and survival.

We also demonstrated in the present series and a previously reported series ${ }^{10}$ that tumor regression and low residual tumor cell density are predictive factors of survival in rectal cancer after preoperative radiotherapy. Several clinical studies on the efficacy of preoperative radiotherapy of rectal cancer correlated survival with the presence or absence of residual tumor in the surgical specimen. ${ }^{26,28}$ In the present series, $79 \%$ of the patients showed histologically partial tumor regression, whereas only $21 \%$ of patients showed no evidence of tumor regression. We found that the TRG strongly correlated with survival and local control. In addition, in contrast with the findings of Berger et al, ${ }^{26}$ we found that residual tumor cell density significantly correlated with DFS. We found not a single case of complete tumor regression after preoperative radiotherapy. Several factors seem to determine tumor regression after preoperative radiotherapy, including fraction size and total dose applied.25,32 In randomized studies with a dose of no more than $20 \mathrm{~Gy}$, no downstaging or complete histological tumor regression was found. ${ }^{24,32} \mathrm{Tu}-$ mor regression was reported with a total radiation dose exceeding 20 Gy. ${ }^{1,3,19,27-30}$ Another factor is the time interval between the end of radiotherapy and surgical intervention. The optimal interval remains a matter of debate. No complete tumor regression has been reported when surgery is performed within a week after the end of radiotherapy, ${ }^{1,17,19}$ a finding consistent with our own data. In contrast, in studies with a longer interval ( $>10$ days), complete histological regression is observed in $2 \%$ to $11 \%$ of patients. ${ }^{15,26,28}$

Based on published data illustrating fast cellular kinetics in rectal cancer, we adopted a short delay in our protocol to counter potential tumor repopulation. ${ }^{37,38} \mathrm{In}$ conclusion, because we are facing locally advanced tumors and a short delay between the end of radiotherapy and surgery, the lack of complete response is not unexpected. However, and most importantly, before a complete tumor regression is diagnosed, the entire tumor region must be sampled and carefully screened to detect any small surviving foci of carcinoma.

This study showed that preoperative radiotherapy decreased local recurrences in locally advanced tumors even if those tumors are fixed. However, the high incidence of distant metastases clearly illustrates the need 
to implement chemotherapy as soon as possible. In this respect, we performed a phase I trial combining CPT-11 and HART. ${ }^{39}$ To date, there is still no evidence for the efficacy of chemotherapy in this setting.

In conclusion, histological assessment of those parameters classically recognized as prognostic factors for nonirradiated colorectal cancer remains valuable for rectal carcinoma treated with preoperative radiotherapy. In addition, pathologists need to pay attention to both the RRM and the clearance, because clearance $<2$ $\mathrm{mm}$ is a very strong indicator of local failure.

\section{REFERENCES}

1. Swedish Rectal Cancer Trial: Local recurrence rate in a randomized multicentre trial of preoperative radiotherapy compared with operation alone in resectable rectal carcinoma. Eur J Surg 162:397-402, 1996

2. Stockholm Colorectal Cancer Study Group: Preoperative short-term radiation therapy in operable rectal carcinoma. A prospective randomized trial. Cancer 66:49-55, 1990

3. Pahlman L, Glimelius B: Pre- and postoperative radiotherapy in rectal and rectosigmoid carcinoma: Report from a randomized multicentre trial. Ann Surg 211:187-195, 1990

4. Medical Research Council Rectal Cancer Working Party: Randomised trial of surgery alone versus radiotherapy followed by surgery for potentially operable locally advanced rectal cancer. Lancet 348:1605-1610, 1996

5. Coucke PA, Sartorelli B, Cuttat JF, et al: The rationale to switch from postoperative hyperfractionated accelerated radiotherapy to preoperative hyperfractionated accelerated radiotherapy in rectal cancer. Int J Rad Oncol Biol Phys 32:181-188, 1995

6. Coucke PA: On behalf of the participants to trial 93-01: Curative resection rate after hyperfractionated accelerated radiotherapy (HART) immediately followed by surgery in locally advanced rectal cancer [abstract]. Radiother Oncol 43:S45, 1997

7. Sobin LH, Wittekind C (eds): IUCC TNM Classification of Malignant Tumors (ed 5). New York, Wiley, 1997

8. World Health Organization: International Histological Classification of Tumours, in Jass RJ, Sobin LH (eds): Histological Typing of Intestinal Tumours (ed 2). Berlin, Springer-Verlag, 1989

9. Mandard AM, Dalibard F, Mandard JC, et al: Pathologic assessment of tumor regression after preoperative chemoradiotherapy of esophageal carcinoma. Clinicopathologic correlations. Cancer 73: 2680-2686, 1994

10. Bouzourene H, Bosman FT, Seelentag W, et al: Importance of tumor regression assessment in predicting the outcome in patients with locally advanced rectal carcinoma who are treated with preoperative radiotherapy. Cancer 94:1121-1130, 2002

11. Quirke P, Durdey P, Dixon MF, et al: Local recurrence of rectal adenocarcinoma due to inadequate surgical resection. Histopathological study of lateral tumour spread and surgical excision. Lancet 1:996-998, 1986

12. MacFarlane JK, Ryall RD, Heald RJ: Mesorectal excision for rectal cancer. Lancet 341:457-460, 1993

13. Dahlberg M, Glimelius B, Pahlman L: Improved survival and reduction in local failure rates after preoperative radiotherapy: Evidence for the generalizability of the results of the Swedish Rectal Cancer Trial. Ann Surg 229:493-497, 1999

14. Kapiteijn E, Marijnen CA, Nagtegaal ID, et al: Preoperative radiotherapy combined with total mesorectal excision for resectable rectal cancer. N Engl J Med 345:638-646, 2001

15. Gerard A, Buyse M, Nordlinger B, et al: Preoperative radiotherapy as adjuvant treatment in rectal cancer. Final results of a randomized study of the European Organization for Research and Treatment of Cancer (EORTC). Ann Surg 208:606-614, 1988

16. Camma C, Giunta M, Fiorica F, et al: Preoperative radiotherapy for resectable rectal cancer: A meta-analysis. JAMA 284:10081015,2000

17. Goldberg PA, Nicholls RJ, Porter NH, et al: Long-term re- sults of a randomised trial of short-course low-dose adjuvant preoperative radiotherapy for rectal cancer: Reduction in local treatment failure. Eur J Cancer 30:1597-1599, 1994

18. Reed WP, Garb JL, Stark AJ, et al: 10-year results of preoperative radiotherapy in treatment of rectal carcinoma. Chirurg 67: 621-624, 1996

19. Stockholm Colorectal Cancer Study Group: Randomized study on preoperative radiotherapy in rectal carcinoma. Ann Surg Oncol 3:423-430, 1996

20. Swedish Rectal Cancer Trial: Improved survival with preoperative radiotherapy in resectable rectal cancer. N Engl J Med 336: 980-987, 1997

21. Petersen S, Hellmich G, Baumann M, et al: Brief preoperative radiotherapy in surgical therapy of rectal carcinoma. Long-term results of a prospective randomized study. Chirurg 69:759-765, 1998

22. Glimelius B, Isacsson U, Jung B, et al: Radiotherapy in addition to radical surgery in rectal cancer: Evidence for a doseresponse effect favoring preoperative treatment. Int J Radiat Oncol Biol Phys 15:281-287, 1997

23. Medical Research Council Rectal Cancer Working Party: Second report. The evaluation of low-dose pre-operative X-ray therapy in the management of operable rectal cancer: Results of a randomly controlled trial. Br J Surg 71:21-25, 1984

24. Willett CG, Badizadegan K, Ancukiewicz M, et al: Prognostic factors in stage T3N0 rectal cancer: Do all patients require postoperative pelvic irradiation and chemotherapy? Dis Colon Rectum 42:167173,1999

25. De Haas-Kock DF, Baeten CG, Jager JJ, et al: Prognostic significance of radial margins of clearance in rectal cancer. Br J Surg 83:781-785, 1996

26. Berger C, de Muret A, Garaud P, et al: Preoperative radiotherapy (RT) for rectal cancer. Predictive factors of tumor downstaging and residual tumor cell density (RTCD): Prognostic implications. Int J Radiat Oncol Biol Phys 37:619-627, 1997

27. Gerard JP, Chapet O, Morignat E, et al: Preoperative radiotherapy of rectal cancer. The Lyons experience, 1985-1996. Prognostic study apropos of 312 patients.. Ann Chir 53:1003-1010, 1999

28. Kaminsky-Forrett MC, Conroy T, Luporsi E, et al: Prognostic implication of downstaging following preoperative radiation for operable T3-T4 rectal cancer. Int J Radiat Oncol Biol Phys 42:935-941, 1998

29. Graf W, Dahlberg M, Osman MM, et al: Short-term preoperative radiotherapy results in down-staging of rectal cancer: A study of 1316 patients. Radiother Oncol 43:133-137, 1997

30. Read TE, McNevin MS, Gross EK, et al: Neoadjuvant therapy for adenocarcinoma of the rectum: Tumor response and acute toxicity. Dis Colon Rectum 44:513-522, 2001

31. Cedermark B, Johansson H, Rutqvist LE, et al: The Stockholm I trial of preoperative short-term radiotherapy in operable rectal carcinoma. A prospective randomized trial. The Stockholm Colorectal Cancer Study Group. Cancer 75:2269-2275, 1995

32. Marijnen CA, Nagtegaal ID, Klein-Kranenbarg E, et al: No downstaging after short-term preoperative radiotherapy in rectal cancer patients. J Clin Oncol 19:1976-1984, 2001

33. Symonds DA, Vickery AL Jr: Mucinous carcinoma of the colon and rectum. Cancer 37:1891-1900, 1976

34. Nagtegaal ID, Marijnen CAM, Kranenbarg EK, et al: Shortterm preoperative radiotherapy interferes with the determination of pathological parameters in rectal cancer. J Pathol 197:20-27, 2002

35. Qizilbash AH: Pathologic studies in colorectal cancer. A guide to the surgical pathology examination of colorectal specimens and review of features of prognostic significance. Pathol Annu 17:1-46, 1982

36. Nagtegaal ID, Marijnen CAM, Kranenbarg EK, et al: Circumferential margin involvement is still an important predictor of local recurrence in rectal carcinoma. Am J Surg Pathol 26:350-357, 2002

37. Rew DA, Wilson GD, Taylor I, et al: Proliferation characteristics of human colorectal carcinomas measured in vivo. Br J Surg 78:60-66, 1991

38. Terry NH, Meistrich ML, Roubein LD, et al: Cellular kinetics in rectal cancer. Br J Cancer 72:435-441, 1995

39. Voelter V, Stupp R, Matter M, : Preoperative hyperfractionated accelerated radiotherapy (HART) and concomitant CPT-11 in advanced rectal carcinoma. A phase I trial [abstract]. Ann Oncol 13(suppl 5):90, 2002 